NOTAS E COMUNICAÇÕES

\title{
BESOUROS DINASTÍNEOS (COLEOPTERA, SCARABAEIDAE, DYNASTINAE) DE QUERARI, MUNICÍPIO DE SÃO GABRIEL DA CACHOEIRA, ESTADO DO AMAZONAS, BRASIL.
}

\author{
Ricardo ANDREAZZE ${ }^{1}$; Catarina da Silva MOTTA ${ }^{2}$
}

\begin{abstract}
RESUMO - Besouros dinastíneos foram coletados em Querari, Município de São Gabriel da Cachoeira, região do alto rio Negro, Estado do Amazonas, de abril a maio de 1993. Utilizaram se lâmpadas de luz mista de mercúrio de $250 \mathrm{~W}$. BL e BLB, sobre um lençol branco, em 33 noites de coletas de 12 horas consecutivas. Foram coletados 76 indivíduos de 20 espécies e 10 gêneros. A tribo Cyclocephalini foi a mais representada (10 espécies), seguida por Phileurini (4 spp.), Oryctini (3 spp.), Dynastini (2 spp.) e Pentodontini (1sp.). Dessas, 7 espécies são registradas pela primeira vez para o Amazonas, 5 delas para o Brasil.
\end{abstract}

Palavras-chave: Coleoptera, Scarabaeidae, Dynastinae, Amazônia brasileira, Querari

Dynastinae Beetles (Coleoptera, Scarabaeidae, Dynastinae) from Querari, District of São Gabriel da Cachoeira, State of Amazonas, Brazil.

ABSTRACT - Dynastinae beetles were collected at Querari, District of São Gabriel da Cachoeira, upper rio Negro, State of Amazonas in April-May/1993. A mixed 250W mercury vapor lamp, a black light (BL) and black light blue (BLB) lamps were used to atract insects on a white sheet in 33 collecting night periods of 12 consecutive hours. 76 specimens were collected, of 20 species, and 10 genera were identified. The tribe Cyclocephalini was the most representative (10 species), followed by Phileurini (4 spp.), Oryctini ( 3 spp.), Dynastini (2 spp.) and Pentodontini (1 sp.). 7 species were recorded for the first time to the State of Amazonas, 5 to Brazil.

Key-words: Coleoptera, Scarabaeidae, Dynastinae, Brazilian Amazon, Querari.

Os besouros dinastíneos representam um grupo peculiar dentre os Scarabaeidae que chegam a apresentar grande tamanho (alguns atingem mais de $20 \mathrm{~cm}$ de comprimento) e estruturas diferenciadas na cabeça e protórax, como os chifres, que os tornam um dos grupos mais exóticos entre os coleópteros (Endrödi, 1985). São comumente chamados de besouroescaravelho, besouro-rinoceronte, besouro-de-chifre e em alguns locais do Brasil são temidos pela sua aparência, barulho que fazem ao voar e crendices que até atribuem malefícios causados por estes insetos (Lenko \& Papavero, 1996).

Uma expedição ao rio Uaupés, região do alto rio Negro, na localidade de Querari, tornou-se um marco para o conhecimento dos dinastíneos dessa região da Amazônia brasileira. Querari $\left(01^{\circ} 05^{\prime} \mathrm{N} / 69^{\circ} 51^{\prime} \mathrm{W}\right)$ é uma vila militar na fronteira com a Colômbia, localizada à margem esquerda do rio

\footnotetext{
${ }^{1}$ Departamento de Identificação e Delimitação (DEID), Fundação Nacional do Índio (FUNAI), SRTVS Qd. 702/902, Plano Piloto 70340-904, Brasília, DF. e-mail: ricardo@funai.gov.br ${ }^{2}$ Coordenação de Pesquisas em Entomologia (CPEN). Instituto Nacional de Pesquisas da Amazônia (INPA), c.p. 478, 69011-970 Manaus, AM, Brasil. e-mail: motta@inpa.gov.br
} 
Uaupés, pertencendo ao Município de São Gabriel da Cachoeira, Estado do Amazonas. Essa área, conhecida estrategicamente como "Cabeça-doCachorro", é predominante de floresta primária com uma população na área constituída principalmente por militares do $2^{\circ}$ Pelotão Especial de Fronteira do Exército e îndios Kubeo.

As coletas foram realizadas em uma das residências da vila militar, onde foi aberto em uma das paredes externas, um lençol branco de $1,40 \mathrm{x}$ $2,20 \mathrm{~m}$ e sobre este, como fonte atrativa, foram colocadas uma luz mista de mercúrio de $250 \mathrm{~W}$, uma lâmpada BL (black-light) e uma BLB (black light blue), as lâmpadas ficaram em posição vertical. Este método já foi utilizado em levantamentos anteriores desses coleópteros, sendo ainda o mais indicado para a captura dos dinastíneos (Andreazze \& Fonseca, 1998; Andreazze, 2001).

Foram realizadas coletas em 33 noites consecutivas em intervalos de 12 horas seguidas (das 18:00 às 06:00h) no período de 06/04 a 22/05/ 1993.

As identificações foram feitas usando-se chaves específicas (Endrödi, 1985 e Lachaume, $1985 ;$ 1992) e pela análise de genitália dos machos. A classificação dos Dynastinae segue a nomenclatura de Lawrence \& Newton (1995). O material foi depositado na

Tabela 1. Espécies de dinastíneos coletados em Querari. Município de São Gabriel da Cachoeira, Estado do Amazonas de 06/04 a 22/05/93. M=machos; $\mathrm{F}$ =fềmeas; inds.=indivíduos; gen.=gẽnero; spp.=espécie. Localidades con asterísco * indicam novos registros.

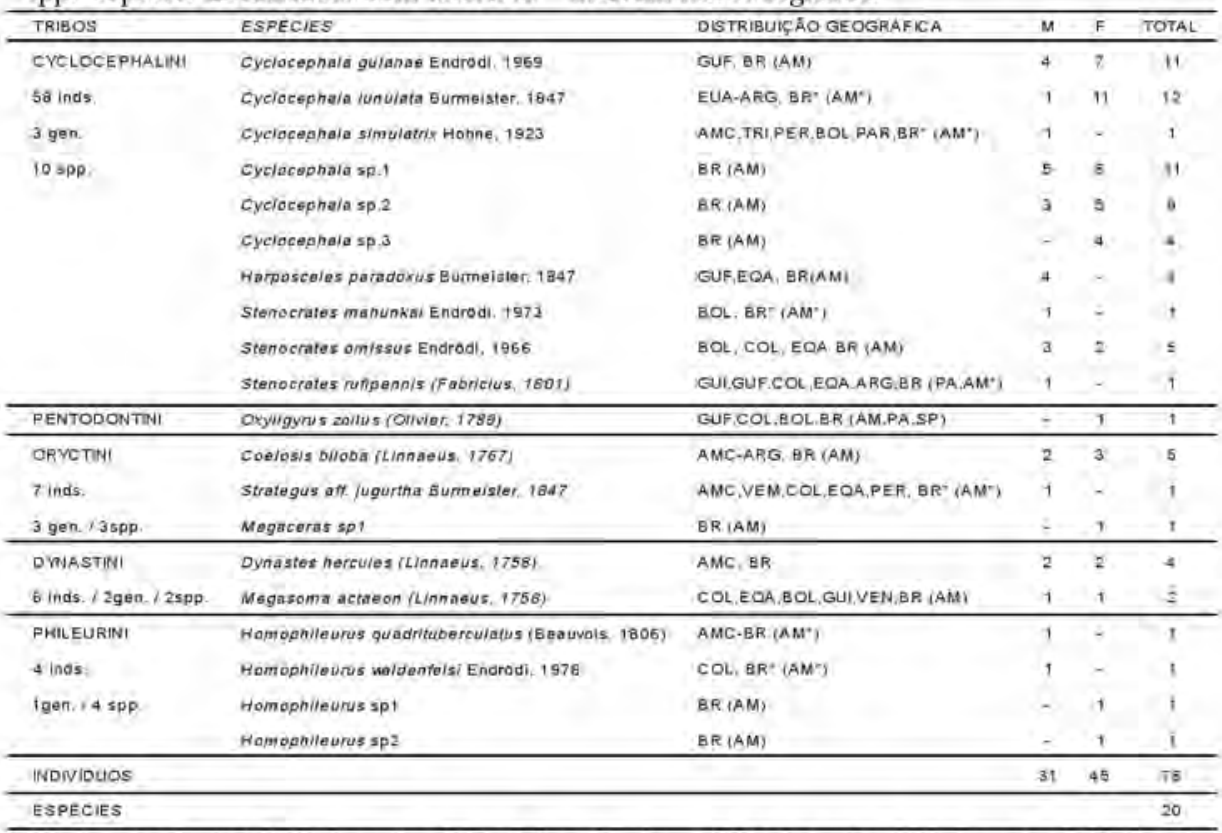

$\mathrm{AMC}=\mathrm{América}$ Central; $\mathrm{ARG}=\mathrm{Argentina} ; \mathrm{BOL}=$ Bolivia; $\mathrm{BR}=\mathrm{Brasil} ; \mathrm{COL}=$ Colombia; $\mathrm{EQA}=\mathrm{Equador} ; \mathrm{EUA}=\mathrm{E}$ stados Unidos da América; GUF=Guiana Francesa; GUI=Guiana; PAR=Paraguaí; PER=Peru; SUR=Suriname; TRI=Trinidad; VEN=Venezuela.

Observação - As siglas separadas por hifen, como por exemplo: EUA-ARG, significa que a espêcie ocorre desde os EUA até a ARG. 
Coleção Entomológica do INPA.

Foram colecionados

76

exemplares de 20 espécies e 10 gêneros, em 33 noites, 396 horas de coletas (Tab.1). A tribo Cyclocephalini foi a mais representativa com 10 espécies distribuidas em 3 gêneros (58 indivíduos, $22 \sigma^{x} / 36$ ㅇ) , seguida por Phileurini com 4 espécies em 1 gênero apenas ( 4 inds., $1 \sigma^{r} / 3$ 우), Oryctini com 3 espécies e 3 gêneros ( 7 inds., $3 \sigma^{\pi} / 4$ 우), Dynastini com 2 espécies e 2 gêneros ( 6 inds., $3 \sigma^{x} / 3$ o) e Pentodontini com 1 única espécie (1우).

Do total, estão sendo registradas pela primeira vez, para o Estado do Amazonas 7 espécies e dentre elas, 5 espécies são novos registros para o Brasil (asssinaladas por asterísco): Cyclocephala lunulata*, Cyclocephala simulatrix*, Stenocrates mahunkai*, Stenocrates rufipennis, Strategus aff. jugurtha*, Homophileurus quadrituberculatus $e$ Homophileurus waldenfelsi*..

O número de espécies capturadas e de registros novos em relação ao número de noites de coleta desta expedição, mostram o quão importante são os levantamentos de coleópteros dinastíneos em áreas da Amazônia de difícil acesso e ainda pouco alteradas.

\section{Agradecimentos}

Ao Comando Militar da Amazônia, Exércio Brasileiro, pelo apoio logístico e assistência aos trabalhos de campo no $2^{\circ}$ Pelotão Especial de Fronteira - Querari. Ao Sr. Roberto Stieger Leite (INPA) e ao Tenente Coronel Aviador Otelo
Guimarães (Força Aérea Brasileira), pela orientação e colaboração para a realização desta expedição.

\section{Bibliografia citada}

Andreazze, R. 2001. Dinastíneos (Coleoptera, Scarabaeidae, Dynastinae) do Parque Nacional do Jaú, Amazonas, Brasil. Acta Amazonica, Manaus (no prelo).

Andreazze, R.; Fonseca, C.R.V. 1998. Dinastíneos (Coleoptera, Scarabaeoidea, Melolonthidae) em uma área de terra firme na Amazônia Central, Brasil. Acta Amazonica 28(1): 59-66.

Endrödi, S. 1985. The Dynastinae of the world. Dr. W. Junk Publishers, Budapest, Hungary, 800p. 56 plates.

Lenko, K.; Papavero, N. 1996. Insetos no Folclore. 2a . ed. São Paulo, Plêiade/ FAPESP, 468p.

Lachaume, G. 1985. Les Coléoptères du Monde. The Beetles of the World. Vol. 5 Dynastini 1 (première partie). Sciences Nat. Venette. France 85 p., 29 pls.

Lachaume, G. 1992. Les Coléoptères $d u$ Monde. The Beetles of the World. Vol.14 Dynastidae américains. Sciences Nat. Venette. France 89 p., 13 pls.

Lawrence, J.F.; Newton Jr., A.F. 1995. Families and subfamilies of Coleoptera (with elected genera, notes, references and data on family-group names). :779-1006. In: Pakaluk, J.; Slipinski, S.A. (eds.): Biology, Phylogeny, and Classification of $\mathrm{CO}^{-}$ leoptera: Papers Celebrating the 80th Birthday of Roy A. Crowson. Museum i Instytut Zoologii PAN, Warszawa.

Aceito para publicação em 19/08/2002 
